Revue de l'Institut des langues et cultures

d'Europe, Amérique, Afrique, Asie et Australie

$20 \mid 2014$

Le conte : d'un art à l'autre

\title{
La figure de la marâtre dans quelques réécritures contemporaines de "Blanche-Neige »
}

The Character of the Stepmother in Some Contemporary Rewritings of "Snow White"

\section{Pascale Auraix-Jonchière}

\section{(2) OpenEdition}

\section{Journals}

Édition électronique

URL : http://journals.openedition.org/ilcea/2787

DOI : 10.4000/ilcea.2787

ISSN : 2101-0609

Éditeur

UGA Éditions/Université Grenoble Alpes

Édition imprimée

ISBN : 978-2-84310-290-5

ISSN : 1639-6073

Référence électronique

Pascale Auraix-Jonchière, « La figure de la marâtre dans quelques réécritures contemporaines de « Blanche-Neige » », ILCEA [En ligne], 20 | 2014, mis en ligne le 08 décembre 2014, consulté le 19 avril 2019. URL : http://journals.openedition.org/ilcea/2787 ; DOI : 10.4000/ilcea.2787

Ce document a été généré automatiquement le 19 avril 2019

(c) ILCEA 


\title{
La figure de la marâtre dans quelques réécritures contemporaines de «Blanche- Neige $»$
}

\author{
The Character of the Stepmother in Some Contemporary Rewritings of "Snow \\ White"
}

Pascale Auraix-Jonchière

1 Bruno Bettelheim consacre un chapitre de son ouvrage, Psychanalyse des contes de fées, au fantasme de la méchante marâtre, figure littéraire qu'il explique par la dissociation symbolique de la mère en deux entités distinctes aux yeux de l'enfant: la bonne mère (celle qui accède à ses désirs) et la mauvaise mère (celle qui résiste, voire châtie) ${ }^{1}$. Cette hypothèse de lecture expliquerait la fréquence du type féminin hautement dévalorisé qui menace l'enfant et ses doubles dans les contes merveilleux, type dont l'exemple le plus connu est certainement la reine du conte des frères Grimm, «Blanche-Neige ».

2 La marâtre serait donc l'incarnation de l'intolérable, ce que pourrait corroborer l'évolution du conte d'une édition à une autre, les frères Grimm tendant à rendre le conflit plus acceptable en remplaçant la mère par la belle-mère. Dans la version de 1812 en effet, la reine, mère de Blanche-Neige, ne meurt pas : le premier paragraphe, qui la présente cousant et rêvant à sa fenêtre, est immédiatement suivi par l'affirmation de sa beauté superlative : «La reine était la plus belle de toute la contrée et très fière de sa beauté » [Die Königin war die schönste im ganzen Land, und gar stolz auf ihre Schönheit] $]^{2}$. Dans les éditions suivantes en revanche, donc dès 1819, celle-ci meurt, remplacée par une autre reine, qui se pare des mêmes attributs : «Un an plus tard le roi se remaria. C'était une belle femme, mais elle était fière et arrogante ${ }^{3}$ » [Uber ein Jahr nahm sich der König eine andere Gemahlin. Es war eine schöne Frau, aber sie war stolz und übermütig]. D'une édition à l'autre, on note que s'opère un transfert lexical: celui de l'adjectif stolz [fière], caractérisant initial de la figure de la mère, qui définit désormais le personnage, nouveau, 
de la belle-mère. On remarque en outre un renforcement simultané de l'écriture, qui redouble le caractère froid et vindicatif de la nouvelle Reine, grâce à l'attribut « übermütig » [arrogante]. Euphémisation et durcissement vont ainsi de pair : la marâtre est le double sombre de la mère, ce que confirme l'expression « méchante femme », qui devient dans le conte l'équivalent quasi-périphrastique $\mathrm{du}$ terme «Stiefmutter» [belle-mère], négativement valorisé.

C'est au traitement de cette figure de substitution, seule restée dans la mémoire collective, que nous allons nous intéresser. Nous nous plaçons dans une perspective qui est celle de la réécriture littéraire, une optique discursive visant à comparer entre eux des textes et à évaluer les fluctuations de sens que leurs variations structurelles et lexicales induisent. En effet, au fil des diverses reconfigurations auxquelles a pu donner lieu «Blanche-Neige », le personnage de la reine marâtre a pris une ampleur considérable, particulièrement exacerbée dans la littérature contemporaine. Concurremment à ce changement de focalisation, le dispositif actanciel évolue: les relations avec le roi (pourtant inexistant au départ), avec le prince ou encore avec le chasseur changent, de façon parfois radicale, modifiant la perspective et le sens profond du conte.

Il est bien sûr impossible dans l'espace de cet article d'envisager l'ensemble, évolutif et fort complexe, de ces configurations narratives. Je m'interrogerai donc d'abord sur l'origine de cette figure, qui semble elle-même procéder en partie de la reprise d'un texte littéraire antérieur bien connu des frères Grimm, la fabella de Psyché et Cupidon dans L'Âne d'or d'Apulée ${ }^{4}$, pour en dégager les traits fondateurs, avant d'envisager quelques propositions contemporaines dans l'optique d'une analyse différentielle: Le Cas BlancheNeige, pièce de théâtre d'Howard Barker ${ }^{5}$, et Miroir, mon amour, film de Siegrid Alnoy ${ }^{6}$.

\section{En amont, la Vénus des Métamorphoses d'Apulée}

Dans le sillage des recherches fondatrices de Heinz Rölleke, les récents travaux de Ute Heidmann et de Jean-Michel Adam ont montré de manière fort précise que les contes des frères Grimm, au-delà de la simple collecte à laquelle fait référence le titre ${ }^{7}$, se fondaient sur la reprise de textes littéraires antérieurs, "se constituant dans un processus très complexe de reconfiguration des formes génériques déjà existantes et d'histoires déjà racontées » (Heidmann, 2012: 10). Or de toute évidence, le « Sneewittchen » des Grimm révèle la présence d'un substrat antique précis, à l'instar de plusieurs des contes les plus connus réécrits par les deux philologues allemands. Les Grimm se réfèrent du reste explicitement à l'histoire de Psyché et Cupidon « en [la] désignant comme "Légende", mais surtout en [la] qualifiant de "Märchen" ", comme l'explique Loreto Núñez (2012: 215) ${ }^{8}$. Or l'histoire de Vénus telle qu'elle est relatée dans le livre IV des Métamorphoses est celle d'une déesse-mère omnipotente et célébrée pour sa beauté, déchue à cause d'une jeune rivale, la pure Psyché: "une nouvelle Vénus, qui possédait encore la fleur de sa virginité » (AO, p. 111). Chez l'auteur latin, le «transfert insolent » qui prive Vénus des honneurs et de l'attention de tous au profit de la jeune fille est à l'origine de la jalousie de la déesse et provoque sa colère : «[...] je ferai en sorte que cette beauté lui donne lieu de se repentir » (AO, p. 112), affirme-t-elle. La marâtre de «Blanche-Neige ", qui « ne pouvait souffrir que quelqu'un puisse la surpasser en beauté » (CPEM, p. 296) [und konnte nicht leiden, daß sie an Schönheit von jemand sollte übertroffen werden], peut être considérée comme un possible avatar de Vénus, une Vénus au miroir obsédée par sa suprématie et son image. Dans ce contexte analogique, le parallèle des scénarios se redouble en outre d'une 
communauté d'expressions. Pour se venger, Vénus choisit son propre fils comme mandataire : «Elle appelle sur-le-champ son fils » pour le sommer de faire de Psyché la plus malheureuse des femmes. Quant à la marâtre du conte, elle donne des ordres à un émissaire pour qu'il la débarrasse de sa rivale : «Un jour, elle appela un chasseur près d'elle» (CPEM, p. 297) ( $D a$ rief sie einen Jäger»). Cet appel commun scelle une incontestable parenté entre la figure de Vénus, belle-mère cruelle dans la suite de la fabella, et la Reine du conte: c'est bien autour de cette rivalité que se noue l'histoire, opposant à une figure volitive et toute-puissante une enfant ou une jeune fille pure.

Dès lors, l'adresse au miroir se révèle essentielle : la rupture de l'énoncé à l'origine du drame repose en effet sur une expansion du discours qui donne place à l'autre là où il n'existait pas auparavant. La formule donnée comme récurrente : «Majesté, vous êtes la plus belle du pays» [ "Frau Königin, Ihr seid die Schönste im Land»], se développe en introduisant un tiers et en posant tout à la fois un parallélisme, une antithèse et une comparaison dévalorisante : «Majesté, vous êtes la plus belle chez nous, / Mais BlancheNeige est mille fois plus belle que vous.» (CPEM, p. 296) [« Frau Königin, Ihr seid die Schönste hier, / aber Sneewittchen ist tausendmal schöner als Ihr »]. L'adjectif «belle» («schön») se répète, qualifiant l'une comme l'autre des protagonistes. Ce même processus était déjà à l'œuvre chez Apulée: dans L'Âne d'or, le nom de "Vénus ", au lieu de désigner la seule Déesse, est également employé pour caractériser la beauté de la jeune Psyché. C'est cette communauté d'expression - cette reprise lexicale ou onomastique - qui, dans les deux cas, met en place le thème de la rivalité. Qui plus est, la formule affirme un changement de statut radical de la Reine dont la beauté, autrefois absolue, n'est plus que relative, tandis qu'à l'inverse Blanche-Neige, auparavant inexistante, accède à un rang de supériorité, faisant dès lors fonction d'usurpatrice.

7 Nombreux sont en outre les épisodes parallèles dans les deux textes. L'obstination de la Reine à poursuivre Blanche-Neige réfugiée chez les sept nains évoque la quête de Vénus pour retrouver Psyché, que sa curiosité a conduite à blesser son amant invisible, Cupidon. C'est d'ailleurs à cette occasion que la déesse se désigne comme la «belle-mère » de la jeune femme (AO, p. 141). À l'issue de la dernière épreuve à laquelle celle-ci est soumise par la déesse en colère, Psyché est victime d'un « sommeil de mort, un sommeil vraiment stygien ", dont la tire sans plus attendre l'Amour revenu à ses côtés. Il ne s'agit pas pour moi de commenter ici cette séquence du sommeil létal, tout à fait essentielle au demeurant ${ }^{9}$; l'important est de noter qu'à l'issue de la fabella, la Déesse assiste aux noces de son fils, Amour, et de Psyché, apaisée par Jupiter. Là, «Vénus, au son d'une belle musique, dansa gracieusement » (AO, p. 151), signe sans doute que celle qui orchestre sur terre la naissance du désir accepte cet hymen et la venue à maturité de son fils, ce jeune Cupidon qui lui "parait toujours un petit garçon» (AO, p.136). Les frères Grimm choisissent pour la Reine un dénouement tout différent: comme on sait, invitée aux noces, elle est forcée « de mettre [des] souliers chauffés à blanc et de danser ainsi, jusqu’à ce qu'elle s'effondre, morte, sur le sol » (CPEM, p. 304). Marâtre obsédée par la jalousie, la Reine de "Blanche-Neige » est vouée à disparaître, dénouement que Bruno Bettelheim interprète comme suit : «Le conte nous dit de façon symbolique que si nous ne réfrénons pas nos passions incontrôlées, elles finiront par nous détruire. Seule la mort de la reine jalouse (l'élimination de toutes les tourmentes externes et internes) peut ouvrir la porte d'un monde heureux. » $(1976: 322)$

8 Au fondement de l'action dans le conte, la Reine occupe toutefois un rôle secondaire par rapport à l'héroïne dont nous suivons l'évolution. Pourtant, les réécritures 
contemporaines de «Blanche-Neige » tendent à étoffer considérablement cette figure de la mère ou de la belle-mère rivale $^{10}$. C'est cette orientation que nous souhaitons interroger, à l'aune de deux exemples récents, littéraire et filmique.

\section{Reconfigurations, reformulations : une question de représentation}

La Reine de «Sneewittchen » n'est pas décrite. Tout au plus est-elle désignée par sa beauté, ce qui laisse le champ libre à la représentation de ce personnage dans les différentes reformulations du conte. Rien d'étonnant à cela : la Reine agit comme force antagoniste, chargée de traits génériques qui font d'elle une figure quasi allégorique. De même "la puissante Vénus " $(A O$, p. 139) n'avait-elle pas besoin d'être décrite dans la fabella d'Apulée. Il va de soi en revanche que le processus de "transmodalisation ${ }^{11}$ " à l'œuvre dans notre corpus: passage du conte au théâtre puis au scénario cinématographique et à sa transcription filmique, autorise voire requiert de plus amples précisions pour donner toute son épaisseur au personnage dont la vocation est dès lors d'être incarné. En même temps, l'un et l'autre genre restent en deçà de la description telle que la pratique le roman, unique support de représentation pour le lecteur.

Dans Le Cas Blanche-Neige, pièce de théâtre d'Howard Barker, c'est dans les paroles échangées par les personnages que la beauté légendaire de la Reine est mentionnée de façon récurrente et superlative. Dès la scène 2 le Roi déclare: "Vous êtes absolument magnifique » (CBN, p. 122) et dans le scénario de Miroir, mon amour, film réalisé par Siegrid Alnoy, la Reine est «splendide ", elle dégage un véritable "rayonnement» (scén., p. 26). Or cette beauté, si elle garde une marge non négligeable d'imprécision, prend des contours plus nets, qui font signe vers la figuration. En réalité, chez Barker, c'est le corps qui s'impose ; on ne saura rien du visage de la reine, à qui Blanche-Neige déclare : «Votre corps a des proportions excellentes» (CBN, p. 123). Un corps à la peau blanche, aux « jambes longues » et « parfaites» $(C B N, \text { p. } 129)^{12}$. Corps qui aimante, également privilégié dans le scénario de Siegrid Alnoy; mais ici, il s'agit plus précisément "d'une Reine brune » (scén., p. 3) qui s'oppose à l'image de ce qu'elle fut autrefois : une reine «blonde, belle, gracieuse et tendre» (p.17) dont on aperçoit l'image par éclipses au gré des rêveries rétrospectives et lourdes d'idéalisation de Blanche-Neige. De ces deux mères, celle qui occupe le centre de la scène est la « femme » (scén., p. 75), « terriblement brune » et "couronnée d'or" (p.78), qui a désormais supplanté la mère. Le contraste chromatique, physique et psychologique traduit cette bipolarité tensionnelle sur laquelle repose en partie le scénario.

11 La marâtre prend donc corps et son âge est précisé : quarante et un ans dans un cas (Barker), cinquante ans environ dans l'autre (Alnoy). Dans le conte, c'est l'âge de BlancheNeige (sept ans) qui provoque une prise de conscience et renvoie au relais et, partant, au conflit des générations. Et c'est ce conflit qui laisse à penser que la Reine est une femme d'âge mûr. On peut considérer les scènes où cette dernière apparait déguisée en « vieille marchande » (CPEM, p. 299) [ «eine alte Krämerin »] comme une allusion détournée à l'âge qui, quoique restant indéterminé, s'avère problématique : "J'AI QUARANTE ET UN ANS ", insiste la reine de Barker ( $C B N$, p. 148) et dans Miroir, mon amour, à l'occasion d'une scène d'intimité avec le Roi, la Reine se révèle «fragile », et donc infiniment réversible en sa 
puissance de signification ${ }^{13}$, et déclare à son époux : « Nous avons vieilli », «L'étreinte de l'âge me fait perdre l'esprit » (scén., p. 86).

Mais le plus important reste sans doute le déplacement qu'opèrent ces deux œuvres d'un archétype à un autre : ce qui domine dans l'un et l'autre cas est en effet l'image - certes malmenée, mais néanmoins résistante - de la femme fatale, syntagme du reste explicite dans le scénario ${ }^{14}$. Dans ce texte préparatoire, la reine arbore des «ongles impeccablement vernis de rouge carmin » (scén., p. 22), elle est « terriblement élégante » ( scén., p. 35) et dans l'obscurité de la chambre apparaît, seule, "la lueur rouge de l'extrémité incandescente d'une cigarette ", « entre [ses] lèvres » (scén., p. 83). Le motif du point rouge reprend dans ce cas précis celui des gouttes de sang sur la neige, image matricielle dans le conte des frères Grimm. Dans le scénario, l'image se dissémine et prend des apparences diverses: ongles, cigarette et plus encore, on va le voir, filet de sang. Dans la voiture qui conduit Blanche-Neige, le Prince et les parents de ce dernier au château de la Reine et du Roi, la mère du Prince, la douce reine Aurore, blonde, blanche et vaporeuse, qui quant à elle n'a jamais quitté son statut de mère, tricotant la trame d'un temps toujours égal, "se pique d'une aiguille » en cousant : "une goutte de sang perle autour de son doigt délicat » (scén., p. 21). Cette image mièvre de la mère rêveuse, rivée à des travaux féminins, associés au foyer, et dont la figure finit d'ailleurs par devenir totalement transparente, est confrontée à son antithèse : la mère de Blanche-Neige, qui elle ne renonce pas à être femme, d'où des scènes récurrentes d'érotisme, exacerbées par un sadomasochisme qui ne laisse pas de marquer le corps. C'est pour cette raison que, par exemple, la Reine confie avec délectation à son soupirant, le Roi Jacob: "J'ai des cicatrices" (scén., p. 62); et de la même façon, quand le miroir se brisant en éclats la blesse à la cuisse, «[d]es perles de sang affluent» (scén., p. 69), rappelant ainsi une séquence antérieure où l'amant chasseur la rhabille et essuie à la bordure de sa robe "une larme de sang" (scén., p. 23). Autre exploitation des "trois gouttes de sang" répandues sur la neige, ces images servent de contrepoint à celle de la cousette « délicate ».

La figure de la femme fatale s'accompagne ainsi de tout un ensemble d'attributs. Le soulier, tout droit issu de la scène finale du conte des Grimm, est fondamental dans ce contexte. En effet les pieds de la Reine cristallisent tout particulièrement l'attention fétichiste que l'on retrouve dans les deux œuvres. Dans la chaumière du Chasseur, «les pieds fins et nus de la Reine chaussent une paire d'escarpins noirs, très noirs, hauts, très hauts » (scén., p. 23), dont le bruit des talons n'aura de cesse de résonner dans les couloirs du château tout au long du film. Chez Howard Barker déjà, la vieille femme portant le miroir de la Reine affirmait : " Majesté / Le son de vos talons dessine votre corps même pour mes yeux d'aveugle » (CBN, p. 132).

14 Dans un cas comme dans l'autre, la chair fait ainsi son entrée dans le conte, qui la tenait à distance.

\section{La marâtre et la séduction}

Parler de marâtre comme nous le faisons ici n'est pas sans poser problème dans des œuvres qui n'utilisent jamais explicitement ce terme. Fidèle en cela aux éditions les plus connues des contes de Grimm, Barker donne voix à « la Reine ", dont on comprend qu'il s'agit de la seconde épouse du Roi, bien que Blanche-Neige l'appelle « ma mère ${ }^{15}$ ». Dans le drame, en effet, il est clairement précisé que la Reine ne peut pas avoir d'enfant. Blanche- 
Neige fait d'ailleurs allusion à celle qu'elle appelle «ma vraie mère », lorsqu'elle évoque un passé révolu. Or la Reine est mise en relation avec la citation que le dramaturge donne en épigraphe : «La méchante marâtre de Blanche-Neige fut elle aussi conviée au festin » ( $C B N$, p. 117), qui renvoie au dénouement du conte. L'important ici est la présence du syntagme " méchante marâtre », qui met en œuvre le phénomène d'« irradiation » tel que le définit Pierre Brunel à propos des mythes ${ }^{16}$. Barker choisit une traduction qui redouble les connotations dépréciatives attachées à la figure de la marâtre et rend saillant le terme que tait le texte.

Il n'en va pas de même dans le scénario de Siegrid Alnoy, qui propose à cet égard une structure plus complexe. Celui-ci présente une figuration triangulaire composée de la reine Aurore, mère du Prince, et de la double représentation, présente et passée, de la Reine. Aurore, femme " au visage très doux et lumineux, vêtue d'étoffes vaporeuses et claires » et aux gestes toujours mesurés, qui semble glisser plus qu'elle ne marche (Aurore Clément dans le téléfilm), se présente d'abord comme une mère idéale. Elle déclare à Blanche-Neige : «Vous serez la fille que je n'ai jamais eue.» (scén., p. 8) Mais, comme on l'a vu, elle finit par devenir insipide et s'effacer pour laisser place à la Reine, cette prétendue mauvaise mère. Le scénario en effet s'ouvre sur un rêve qui rejoue la scène de l'abandon dans la forêt par le chasseur. Or la figure de la Reine s'y surimprime de deux façons : par sa voix - une voix off qui reprend quasi textuellement les paroles de la marâtre dans l'hypotexte ${ }^{17}$; puis par un plan en plein cadre qui la représente en cannibale qui «se repaît à pleines mains d'aliments sanguinolents " (scén., p. 3), plan particulièrement saisissant, qui illustre une esthétique de la cruauté. Ces premières séquences introduisent le téléspectateur dans le conte des Grimm de façon abrupte et violente. Dans la mémoire collective, cette femme surpuissante qui domine la scène et l'écrase de son imposante présence absente ne peut être que la marâtre épouvantable de "Sneewittchen ». Pourtant, le scénario (volontairement ou non) renoue avec la version de 1812 : la Reine est bien la mère biologique de Blanche-Neige. Mais le plus frappant est le fait que celle-ci se dédouble dans les souvenirs de la jeune Princesse, comme on l'a vu. Le film fait alors alterner les images antithétiques, brouillées par l'eau du miroir dans laquelle elles se reflètent, des deux mères : celle de l'enfance et celle de l'adolescence, ce qui semble correspondre à une vision intérieure, éminemment subjective. Dans ces séquences mémorielles, Blanche-Neige prend d'ailleurs l'apparence d'une petite fille. Par contraste, la mère actuelle, cette "mère épouvantable (scén., p. 15), est qualifiée de " féroce » par un glissement de sens qui lui confère une dimension animale et l'apparente à ces « bêtes sauvages » dont le chasseur craint qu'elles ne dévorent l'enfant dans la forêt.

17 Ainsi la Reine, devenue femme fatale, reste la rivale de Blanche-Neige en incarnant une féminité superlative, mais ce choix de représentation déplace le problème. En effet celleci ne cherche plus à éliminer la jeune fille, dont elle ne redoute pas la beauté. Dans Le Cas Blanche-Neige, son propre charme est d'ailleurs écrasant, lié à une attraction érotique qui s'exerce aussi bien sur le serviteur que l'on surprend en pleine forêt, la Reine étant nue, dans une scène particulièrement crue, à l'ouverture de la pièce, que sur « le roi de tous les Irlandais ", père du Prince, ou sur le Prince lui-même. De la même façon dans Miroir, mon amour, la Reine séduit le Roi Jacob, avec qui elle partage de longue date une complicité amoureuse, le Prince pendant un temps, le Chasseur, et le jardinier. L'ascendant du charme de la Reine résiste à la force potentiellement destructrice de l'âge. Malgré les sarcasmes de Blanche-Neige, hommage est rendu à la beauté de cette femme par ses amants ou par ses soupirants ${ }^{18}$. On est donc loin de la colère et de la frayeur qui 
s'emparent tour à tour de la marâtre des Grimm à la seule annonce de l'entrée de Blanche-Neige dans l'âge de la séduction. Dans ces nouvelles représentations, c'est la jeune fille qui cherche à éliminer sa rivale et non l'inverse.

18 Certes, la mise à mort n'est pas effective, mais l'intention reste transparente : les mots sont des armes redoutables et la Princesse en use avec violence: "Vous exhibez votre corps dans ces vêtements moulants alors qu'en réalité vous êtes plutôt vieille ", déclare-telle dans la pièce de Barker ( $C B N$, p. 123). Blanche-Neige répand ses propos venimeux, tentant de convaincre son propre père ou les amants de la Reine de la dégradation de celle-ci. Ainsi, avec le serviteur : «Je suis plus belle que la reine ma mère : ses seins sont tout ratatinés » (p. 131). Et la Blanche-Neige névrosée de Miroir, mon amour d'assaillir sa mère d'invectives: «Ces robes ne sont plus taillées pour vous! Vous exhibez un corps vieilli ! Votre coquetterie est absurde. » (Scén., p. 73) C'est donc Blanche-Neige qui ne parvient pas à trouver sa place aux côtés d'une mère ou d'une belle-mère qu'elle imagine en redoutable marâtre. Le Prince explique à son père le Roi Jacob que Blanche-Neige est « terrorisée " par sa mère : « Il lui arrive même de rêver que sa mère veut la tuer. » (Scén., p.11) Les séquences inaugurales du film mettent en images des scènes érotiques et cruelles, dominées par la figure souveraine de la Reine maléfique. Mais Blanche-Neige dort, elle rêve. Le fantasme puise à la source des contes qui, en l'occurrence, ne coïncident guère avec la réalité : si en effet la Reine demande au Chasseur de verser le sang, c'est le sien propre, que l'on voit perler sur son cou, dans une relation de plaisir masochiste où la lacération devient le signe d'une soumission volontaire.

Cette inversion repose en partie sur la forte relation œdipienne que ces Blanche-Neige, plus sournoises que vraiment blanches, entretiennent avec leur père. Au Prince qui l'interroge la jeune fille répond : « Oh, avec mon père, tout est simple : il m'adore. On est très proches... » (scén., p. 16). La séquence la plus éloquente du film à cet égard est celle où la Princesse demande au Roi de lui masser le ventre "comme autrefois ». Le scénario explique : « la main de Blanche Neige se saisit doucement de celle de son père, et la fait passer sous son vêtement » (scén., p. 43) ; en réalité, on voit la jeune fille conduire la main du Roi vers son sexe. Dans Le Cas Blanche-Neige, où le corps de la femme se métaphorise en paysage, «à l'inverse de Peau d'Âne, [la jeune fille] multiplie, dans sa hâte d'accéder au savoir, les invitations à l'inceste: "Cher papa venez voir mon jardin"19". Cette relation œdipienne trahit l'immaturité de Blanche-Neige et son malaise devant une sexualité assumée. Bien qu'elle multiplie elle-même les tentatives de séduction, celles-ci avortent les unes après les autres. Une séquence filmique la montre simulant une tentative de viol de la part du Chasseur : elle se dénude, corps blanc sur fond de forêt sauvage, s'empare de la dague qu'elle retourne contre elle, mime la menace. Mais le Chasseur reprend son arme et la replace à sa ceinture avant de rhabiller la jeune fille, démontrant son insensibilité aux charmes de la jeunesse, et son faible pour une maturité sexuelle à ses yeux plus savoureuse. On trouve une scène analogue avec le serviteur dans la pièce de Barker. Blanche-Neige, nue, essaie de convaincre, mais en vain, l'amant récalcitrant de la Reine de sa plus-value érotique :

\section{SERVITEUR}

$-[. .$.$] il se peut que la fille de votre mère me fasse un effet considérable et il se peut$ bien que le Roi de tous les Irlandais voie juste en ce qui concerne vos jambes [...] donc il se peut qu'il se passe quelque chose mais certainement pas la même chose pas même quelque chose d'approchant je crois encore moins exactement la même chose (un temps) Mademoiselle. (CBN, p. 130) 
fer », les «tenailles », les "souliers rougeoyants», entrent en résonance avec ce "théatre de la Catastrophe » (Angel-Perez, 2006), ce que souligne spécialement la dernière phrase de l'épigraphe : « Puis on la força à chausser ces souliers rougeoyants, et à danser jusqu'à tomber raide morte » (CPEM, p. 117). Pour Barker, la Reine s'impose jusqu'à la fin en choisissant: connaissant le sort qui l'attend, elle refuse de prendre la fuite; sans doute faut-il qu'elle joue son rôle jusqu'au bout et qu'elle transmette l'image d'un amour teinté de mort. Lors des noces, le Roi lui intime de faire ce qu'il croit être son devoir ${ }^{23}$ : danser la danse de la mort pour mettre à nu les pulsions du sexe.

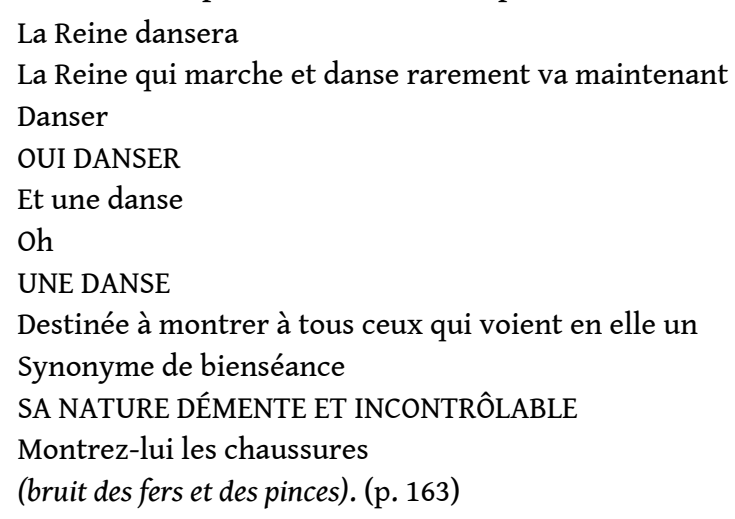

Mais ces chaussures qui font la femme et sa puissance ne seront pas le vulgaire instrument d'un dévoilement des pulsions. Au contraire, la Reine marche, mais ne danse pas; elle se tait, et ne crie pas. L'intensité de sa douleur dépasse toute sensation physique : la Reine avorte, et cette marche sacre la puissance de la femme stérile, qui peut dès lors baptiser Blanche-Neige de ce sang perdu : «Voyez le sang éclatant de son [celle du Roi] ingéniosité / Il resplendit sur vous Blanche-Neige vous désormais / moins bien 
moins blanche que moi » (p. 168). La jeune fille naît ainsi à l'expérience de la douleur et de l'atrocité de l'amour.

Dans Miroir, mon amour, on assiste au contraire à une sorte de passation des pouvoirs, qui repose sur la négation de la rivalité légendaire entre la mère et la fille. «Maman, ma douleur est-elle votre plaisir secret?», demande Blanche-Neige (scén., p. 75), à quoi la Reine répond : «Quel mélo!» (scén., p. 76). Cette mise à distance du conte ouvre la porte à une réécriture qui redéploie les motifs emblématiques de la lutte entre la mère et la fille chez les Grimm : le lacet, la pomme, le miroir. Le scénario souligne la valeur érotique du lacet : avant de ceindre la fille, il corsète la mère. Et c'est Blanche-Neige elle-même qui réclame "un lacet multicolore pour marquer davantage [sa] taille fine" (scén., p. 77). C'est pour mettre en valeur sa féminité qu'elle exige de la Reine qu'elle la «serre davantage ». Le miroir devient dès lors le lieu du transfert : tandis que «l'image de la Reine quitte le miroir » (p. 78), la jeune fille se mire et voit se refléter la scène qui, dans le conte, génère la jalousie et inverse sa leçon. « Miroir, miroir joli, qui est la plus belle en ce royaume?", demande le "reflet de la Reine », et la "Voix de Blanche Neige » répond: « Dame la Reine, vous êtes belle, mais Blanche Neige l'est mille fois plus que vous!» (scén., p. 78). De cette investiture assumée par le bruit des talons de la Reine, qui décroît alors qu'elle cède sa place, témoigne chez Blanche-Neige le remplacement de ses «petites sandales » (p. 78) par « une paire d'escarpins noirs, très noirs », et les échos de son pas " assuré » (ibid.). Grâce à ces chaussures, elle séduira le jardinier, autrefois insensible, et son Prince, éveillé à la jalousie et aux tourments du désir. Quant à la pomme, croquée par les amants dans le verger, elle se démultiplie dans le panier débordant que le jardinier renverse sur la jeune femme abandonnée sur le sol et se métaphorise pour dire la pâmoison amoureuse : Blanche-Neige découvrant le plaisir "tombe dans les pommes ", dira plaisamment le Prince (p. 85).

Certes, au triomphe de la jeune fille correspond la douleur de la Reine, qui «se démaquille, en effaçant des larmes qui glissent le long de ses joues » (scén., p. 85) devant ses miroirs. Miroirs de vérité, qui la montrent vulnérable. Mais le dénouement ouvre de nouvelles perspectives en scellant l'amour complice du Roi et de la Reine. D'elle à lui, pendant le bal, un baiser est délicatement envoyé. Le film s'achève sur ce lien d'amour et un " fondu au blanc ", aux antipodes du baptême sanglant qui clôture la pièce de Barker.

La figure de la marâtre tend à devenir centrale dans les réécritures contemporaines, recentrage qui s'accompagne souvent d'une inversion: elle s'impose comme figure érotique, redéfinissant dans son sillage les rôles et les fonctions des personnages masculins du conte. Et si elle reste une rivale, c'est aux yeux de Blanche-Neige : le conte sert alors de référence pour donner lieu au redéploiement complexe d'un théâtre intérieur dont les diverses configurations renvoient aussi bien aux conflits générationnels qu'à la notion de féminité dans ses diverses implications.

\section{BIBLIOGRAPHIE}

ALNOY Siegrid, réalisatrice (2012), Miroir, mon amour, diffusé sur Arte (17 octobre 2012). 
ANGEL-PEREZ Élisabeth (2002), « Chants d'expérience », H. Barker (dir.), Le Cas Blanche-Neige, Euvres choisies (vol. 4, 9-12), Montreuil-sous-Bois : éditions théâtrales, Maison Antoine Vitez. ANGEL-PEREZ Élisabeth (2006), Howard Barker et le théâtre de la Catastrophe, Montreuil-sous-Bois : éditions Théâtrales.

APULÉE, L'Âne d'or ou les métamorphoses, traduit du latin par P. Grimal (1985), Paris : Gallimard, Folio.

BARKER Howard (2002), Le Cas Blanche-Neige, Comment le savoir vient aux jeunes filles, [Knowledge and a girl (The snow white case)], traduit de l'anglais par C. Menon (2002), CEuvres choisies (vol. 4), Montreuil-sous-Bois : éditions théâtrales, Maison Antoine Vitez.

BetTelheim Bruno (1976), La Psychanalyse des contes de fées, traduit de l'américain par T. Carlier (1976), Paris : Laffont, 2012.

BRUNEL Pierre (1992), Mythocritique. Théorie et parcours, Paris : PUF écriture.

GENETTE Gérard (1982), Palimpsestes, Paris : Seuil, « Points ».

GRIMM Jacob et Wilhelm, Kinder- und Hausmärchen ( $1^{\text {re }}$ éd. 1857, 3 vol.), [Contes pour les enfants et la maison], Ausgabe letzter Hand mit den Originalanmerkungen der Brüder Grimm, mit einem Anhang sämtlicher, nicht in allen Auflagen veröffentlichter Märchen und Herkunftsnachweisen herausgegeben von Heinz Rölleke [Dernière édition révisée par les frères Grimm, avec une annexe réunissant tous les contes n'ayant pas été publiés dans l'ensemble des éditions, et accompagnée de notes sur l'origine des contes, éditée par Heinz Rölleke] (1993), Stuttgart : Philipp Reclam Jun.

GRIMM Jacob et Wilhelm, Contes pour les enfants et la maison (1857, $1^{\text {re }}$ éd. 1812-1815), collectés par les frères Grimm ; édités et traduits par Natacha Rimasson-Fertin (2009), tomes 1 et 2, Paris : éditions José Corti.

HEIDMANN Ute (2012), « Le dialogisme intertextuel des contes des Grimm », Féeries, 9.

NÚÑEZ Loreto (2012), « Commentaires paratextuels des Kinder-und Hausmärchen », Féeries, 9.

\section{NOTES}

1. Loin d'être un artifice utilisé seulement dans les contes de fées, ce dédoublement de personnalité qui permet à l'enfant de garder intacte l'image favorable, serait utilisé par beaucoup d'enfants pour apporter une solution à un problème de relation trop difficile pour qu'il puisse le régler ou le comprendre.

2. <www.grimms.de/contenido/cms/upload/khmdb/hex/hex272.jpg> (consulté le 22/04/2012), p. 238. Je traduis.

3. Contes pour les enfants et la maison. Collectés par les frères Grimm, édités et traduits de l'allemand par N. Rimasson-Fertin, Paris, José Corti, 2009, vol. I, p. 296 (les citations en français renverront désormais à cette édition et j'abrègerai ce titre en CPEM). Il s'agit de l'édition de 1857. La modification, toutefois, existe dès l'édition de 1819. Pour la version en allemand, nous renvoyons à l'édition de H. Rölleke, Brüder Grimm, Kinder- und Hausmärchen, Stuttgart, Philipp Reclam (1980), 1999, p. 269-278, Sneewittchen.

4. Apulée, L'Âne d'or ou les métamorphoses, trad. du latin par P. Grimal, Paris, Gallimard, Folio, 1985. Désormais j'abrègerai ce titre en $A O$. 
5. H. Barker, Le cas Blanche-Neige, Comment le savoir vient aux jeunes filles [2002, Knowledge and a girl (The snow white case)], trad. de l'anglais par C. Menon, CEuvres choisies, vol. 4, Montreuil-sousBois, éditions théâtrales, Maison Antoine Vitez, 2002. J'abrègerai désormais ce titre en CBN.

6. 2012, diffusé sur Arte le 17/10/2012 (nous remercions vivement S. Alnoy de nous avoir transmis le scénario du film auquel nous renverrons précisément, indiquant les pages entre parenthèses, sous l'abréviation scén.).

7. Kinder- und Hausmärchen gesammelt durch die Brüder Grimm.

8. L. Núñez explique que « dans la partie de la section sur la littérature portant sur d'Aulnoy, les Grimm se réfèrent d'abord à la "légende d'Amour et Psyché" " (KHM, 1856, p. 304 / Rölleke, KHM, 2010, III, p. 329) «puis au "conte d'Amour et Psyché" ». Elle ajoute que «dans l'annotation du conte $\mathrm{n}^{\circ} 88$, "Das singende springende Löweneckerchen », il y a tout un développement sur le "si fameux conte de Psyché” " (ibid., note 50).

9. Le « sommeil stygien » assaille Psyché et «à l'endroit même où elle se tient, sur le sentier, la fait tomber et la tient en son pouvoir » (AO, p. 149). Ainsi Blanche-Neige, ayant croqué la pomme empoisonnée, « tomba morte sur le sol» (CPEM, p. 302).

10. Le tournant s'amorce à la fin du $\mathrm{XIX}^{\mathrm{e}}$ siècle, où le conte de J. Lorrain, La Princesse Neigefleur (1894), est tout entier centré sur la Reine. En 1901, le dramolet de R. Walser accorde un rôle essentiel au personnage, aux côtés du Prince, du Roi et du Chasseur. Tout au long du $\mathrm{xx}^{\mathrm{e}}$ siècle la figure de la Reine s'impose, tant dans le domaine de la littérature que dans celui du cinéma. Les créations les plus récentes confirment et accentuent cette tendance de façon frappante.

11. Genette (1982: 395) désigne ainsi «toute espèce de modification apportée au mode de représentation caractéristique de l'hypotexte».

12. Et p. 153, le Roi admire le talon qui fait ressortir «sa jambe parfaite / Qui fait s'incliner sa hanche parfaite ». Voir la préface p. 12.

13. À l'opposé de la Reine inflexible des frères Grimm, habitée par l'envie, la colère ou la frayeur.

14. «Finalement ", s'insurge Blanche-Neige, «je ne sais pas ce qui est le pire : quand vous jouiez aux mères au foyer, ou maintenant, quand vous jouez aux femmes fatales! (scén., p. 75).

15. Voir par exemple p. 120.

16. «[...] une image mythique, présente dans un texte de [tel] écrivain, peut rayonner dans un autre texte où elle n'est pas explicite » (Brunel, $1992: 84$ ). N. Rimasson-Fertin traduit, fidèle en cela à la lettre du texte, « la marâtre impie » (KHM, p. 304).

17. «Je ne veux plus la voir devant mes yeux! Tu la tueras au loin dans la forêt et tu me rapporteras son foie et ses poumons en témoignage. » (Scén., p. 1)

18. Pour le Chasseur, « un corps qui a vécu » est "émouvant " (scén., p. 47). Pour le serviteur de Barker, à qui l'on révèle l'âge de la Reine : «Sa beauté est intacte quel que soit son âge. » (CBN, p. 141)

19. Angel-Perez (2002, préface : 12). La citation incluse se trouve p. 121.

20. «BLANCHE-NEIGE. - Vous êtes magnifique et abjecte et belle et terrible et votre cadavre planera au-dessus de mon existence entière comme l'ombre de quelque animal aux longs membres. » (p. 158)

21. "Cet enfant est ma mort / Oh ne voyez-vous pas / Que le seul homme au monde dont l'acte d'amour pouvait rendre une grossesse fatale pour moi/ Est votre futur époux? SINON POURQUOI CELA A-T-IL PRIS TANT DE TEMPS ?» (p. 159)

22. Il importe ici de convoquer le sous-titre: Comment le savoir vient aux jeunes filles.

23. «POUVEZ-VOUS FAIRE CELA / POUVEZ-VOUS FAIRE CE QUE VOUS DEVEZ FAIRE » (p. 161). Et encore : « Oui / Vous êtes une légende et il ne faut qu'une légende » (ibid.). 


\section{RÉSUMÉS}

La marâtre de Blanche-Neige procède en partie de la fabella de Psyché et Cupidon dans Les Métamorphoses d'Apulée : rivalité féminine et jalousie sont le moteur du conte chez l'auteur latin comme chez les frères Grimm. Mais dans les réécritures contemporaines de cette histoire célèbre, la Reine tend à devenir une figure centrale, qui a l'étoffe d'un véritable personnage (surtout s'il s'agit de l'incarner sur scène ou au cinéma), et son traitement fait parfois l'objet d'une inversion, comme dans les deux œuvres que nous abordons ici : Le Cas Blanche-Neige, pièce de théâtre d'Howard Barker et Miroir, mon amour, film de Siegrid Alnoy. Critiquée par sa fille qui peine à devenir femme, la Reine est à la fois l'initiatrice (parfois funeste) et l'initiée, obligée de reconsidérer son propre rapport à l'amour.

Snowhite's cruel stepmother partly proceeds from the fabella of Psyche and Cupido in Apulée's Metamorphosis: the tale deals with feminine rivalry and jealousy in both texts. But in the contemporary rewritings of this famous story, the Queen tends to become one of the main characters (mostly when the text is written to be performed on the stage or on the screen). The role she plays is sometimes utterly changed, which is the case in both texts we analyse: $\mathrm{H}$. Barker's play Knowledge and a girl (the Snowhite case) and S. Alnoy's film Miroir, mon amour. The queen is then criticized by her daughter who struggles to become a woman, and she is at the same time the one that guides and teaches (even in a cruel way) and the one that learns how to change her own idea of love.

INDEX

Mots-clés : Blanche-Neige, conte, marâtre, réécriture, transmodalisation, intermédialité Keywords : Snow-white, tale, stepmother, rewriting, transmodalisation, intermediality

\section{AUTEUR}

\section{PASCALE AURAIX-JONCHIÈRE}

Université Blaise Pascal, Clermont-Ferrand, CELIS 